\title{
Why the High-Energy Optical Potential Can Be and Should Be Considered Local*
}

\author{
J. F. READING $\dagger$ \\ Northeastern University, Boston, Massachusetts 02115 \\ (Received 23 October 1969)
}

\begin{abstract}
The approximate treatment of the nonlocality of the optical potential describing scattering from a set of $N$ nonoverlapping potentials is shown to lead to erroneous effective local potentials. The difficulty is circumvented by constructing a local two-body $t$ matrix which leads directly to a local potential.
\end{abstract}

\section{INTRODUCTION}

$\mathbf{T}$ HE scattering from a set of $N$ nonoverlapping potentials $v\left(\mathbf{r}-\mathbf{r}_{i}\right)$ is completely determined by the single potential phase shifts ${ }^{1}$ or by the on-shell $t$ matrix $t\left(E, \mathbf{k}_{E}{ }^{\prime}, \mathbf{k}_{E}\right)$. The optical potential describing scattering from $N$ such potentials ${ }^{2}$ moving with wave functions $\varphi_{i}\left(\mathbf{r}_{i}\right)$ is given to order $N^{-1}$ by

$$
\begin{gathered}
V_{\text {opt }}\left(\mathbf{k}, \mathbf{k}_{0}\right)=N t\left(E, \mathbf{k}, \mathbf{k}_{0}\right) F\left(\mathbf{k}-\mathbf{k}_{0}\right), \\
F(q)=N^{-1} \sum_{i=1}^{N} \exp (-i \mathbf{q} \cdot \mathbf{r})\left|\varphi_{i}(\boldsymbol{r})\right|{ }^{2} d^{3} \boldsymbol{r} \\
=\int \exp (-i \mathbf{q} \cdot \mathbf{r}) \rho(r) d^{3} r .
\end{gathered}
$$

To obtain $V_{\text {opt }}\left(\mathbf{k}, \mathbf{k}_{0}\right)$ in configuration space it is necessary to know $t\left(E, \mathbf{k}, \mathbf{k}_{0}\right)$ off the energy shell, i.e., when

$$
|\mathbf{k}| \neq\left|\mathbf{k}_{0}\right| \neq k_{E}=\left(2 m E / \hbar^{2}\right)^{1 / 2}
$$

However, the scattering will be independent of this off-shell behavior if we do the calculation properly. Unfortunately, the off-energy-shell extrapolation leads to a nonlocality in the optical potential, since in general $t\left(E, \mathbf{k}, \mathbf{k}_{0}\right)$ is not only a function of $\left|\mathbf{k}-\mathbf{k}_{0}\right|$ but $\left|\mathbf{k}+\mathbf{k}_{0}\right|$. This nonlocality is usually treated approximately ${ }^{3}$ and an effective local potential is derived which depends on the off-shell energy behavior of $t$ through the derivative $t^{\prime}\left(E, \mathbf{k}_{E}, \mathbf{k}_{E}\right)$, where

$$
t^{\prime}(E, \mathbf{k}, \mathbf{k})=(\partial / \partial k) t(E, \mathbf{k}, \mathbf{k}) .
$$

In this paper we indicate that this approximation procedure destroys the property of independence of the scattering on the off-shell behavior of $t$ and is therefore inappropriate to this problem. We remove this difficulty by constructing a local two-body $t$ matrix rendering approximations unnecessary. The optical potential is local.

\section{NONLOCALITY OF OPTICAL POTENTIAL}

The nonlocality of the optical potential is measured by the value of $t^{\prime}\left(E, \mathbf{k}_{E}, \mathbf{k}_{E}\right) .^{3}$ In this section we show that there is a wide range for $t^{\prime}\left(E, \mathbf{k}_{E}, \mathbf{k}_{E}\right)$, even though $t\left(E, \mathbf{k}_{E}, \mathbf{k}_{E}\right)$ is fixed.

The wave function $\chi$ describing the scattering from a single potential $v(r)$ is given by

$$
\begin{aligned}
\chi\left(\mathbf{k}_{E}, \mathbf{r}\right)= & \exp \left(i \mathbf{k}_{E} \cdot \mathbf{r}\right) \\
& -\frac{1}{2 \pi^{2}} \int \frac{\exp \left(-i \mathbf{k}^{\prime} \cdot \mathbf{r}\right) t\left(E, \mathbf{k}^{\prime}, \mathbf{k}_{E}\right)}{k_{E}{ }^{2}-k^{2}+i \epsilon} d^{3} k^{\prime} .
\end{aligned}
$$

This equation for $\chi(r)$ involves $t$ off the energy shell because Eq. (4) determines $\chi$ inside the potential region which we will denote by $r<a$.

It is well-known that there are many potentials which will give a wave function $\psi$, equal to $\chi$ for $r>a$, but different from $\chi$ for $r<a$. All will have the same $t$ matrix on shell. It is intuitively obvious that $t^{\prime}\left(E, \mathbf{k}_{E}, \mathbf{k}_{E}\right)$ will differ for each of these and that an effective local potential which is just a linear function of the number $t^{\prime}$ will lead to different answers for the scattering, contrary to the known exact solution of the problem. If the reader is convinced that this is a difficulty, he may skip to Sec. III. The rest of this section is devoted to constructing $t$ matrices which illustrate how bad this can be.

From Watson ${ }^{4}$ we have the equality

$$
\frac{2}{\pi i} \int_{0}^{\infty} \frac{j_{l}\left(k^{\prime} r\right) j_{m}\left(k^{\prime} r^{\prime}\right) k^{\prime 2} d k^{\prime}}{k_{E}{ }^{2}-k^{\prime 2}+i \epsilon}=j_{m}\left(k_{E} r^{\prime}\right) j_{l}\left(k_{E} r\right),
$$

where

$$
m-l=\text { even integer }>0 \quad \text { and } \quad r>r^{\prime}>0 .
$$

With Eq. (5) in mind, we define a $t$ matrix $\tau$ such that

$$
\begin{aligned}
\boldsymbol{\tau}\left(E, \mathbf{k}, \mathbf{k}_{0}\right)= & \sum_{l \text { even }, \text { odd }}(2 l+1) P_{l}\left(\hat{k} \cdot \hat{k}_{0}\right) t_{l}\left(k_{E}, k_{E}\right) \\
& \times \int_{0}^{a} j_{m} \pm(k r) \varphi_{E}(r) j_{m} \pm\left(k_{0} r\right) r^{2} d r / \int_{0}^{a} j_{m} \pm\left(k_{E} r\right) \varphi_{E}(r) j_{m} \pm\left(k_{E} r\right) r^{2} d r .
\end{aligned}
$$

\footnotetext{
* Work supported in part by the National Science Foundation.

$\dagger$ Part of this work was carried out while the author was a fellow at the United Kingdom Atomic Energy Research Establishment, Harwell, England.

${ }_{1}$ See L. H. Schick [Rev. Mod. Phys. 33, 608 (1961)] for a review and earlier references.

${ }^{2}$ K. M. Watson, Phys. Rev. 89, 575 (1953); Rev. Mod. Phys. 30, 565 (1958).

${ }^{3}$ See, e.g., B. Mulligan, Ann. Phys. (N.Y.) 26, 159 (1964); J. F. Reading, Phys. Rev. 156, 1116 (1967). Here it is shown that an effective local potential $V_{e}(r)$ is given by $V_{e}(r)=-2 \pi h^{2} m^{-1} \rho(r) \times N t\left(E, \mathbf{k}_{B}, \mathbf{k}_{E}\right)\left[1-2 \pi N \rho(r) t^{\prime}\left(E, \mathbf{k}_{E}, \mathbf{k}_{E}\right)\right]^{-1}$.

${ }^{4}$ G. N. Watson, Theory of Bessel Functions (Cambridge University Press, New York, 1952), 2nd ed., p. 429.
} 
In Eq. (6), $\varphi_{E}(r)$ is some function zero for $r>a$ and $m^{ \pm}$is an even (odd) integer taken to be $m^{+}\left(m^{-}\right)$if $l$ is even (odd). If we take $m^{ \pm}$to be greater than the maximum $l$ value important in the scattering, we can now use Eq. (5) to show that

(i) $\psi\left(\mathbf{k}_{E}, \mathbf{r}\right)$, given by

$$
\begin{aligned}
\psi\left(\mathbf{k}_{E}, \mathbf{r}\right)= & \exp \left(i \mathbf{k}_{E} \cdot \mathbf{r}\right) \\
& -\frac{1}{2 \pi^{2}} \int \frac{\exp \left(-i \mathbf{k}^{\prime} \cdot \mathbf{r}\right) \tau\left(E, \mathbf{k}^{\prime}, \mathbf{k}_{E}\right)}{k_{E}{ }^{2}-k^{\prime 2}+i \epsilon} d^{3} k^{\prime},
\end{aligned}
$$

has the same form for $r>a$ as does $\chi\left(\mathbf{k}_{E}, \mathbf{r}\right)$, i.e., $\boldsymbol{\tau}\left(E, \mathbf{k}_{E}{ }^{\prime}, \mathbf{k}_{E}\right)=t\left(E, \mathbf{k}_{E}{ }^{\prime}, \mathbf{k}_{E}\right)$;

(ii) the nonlocal optical potential $V_{\tau}$ is

$$
V_{\boldsymbol{\tau}}=N F\left(\mathbf{k}-\mathbf{k}_{0}\right) \tau\left(E, \mathbf{k}, \mathbf{k}_{0}\right) ;
$$

(iii) by choosing $m^{ \pm}$to be asymptotically large we can find an effective local potential through $\tau^{\prime}$, where

$$
\tau^{\prime}\left(E, \mathbf{k}_{E}, \mathbf{k}_{E}\right)=2 \sum_{l \text { even,odd }} m^{ \pm} t_{l}\left(k_{E}, k_{E}\right)(2 l+1) .
$$

Now, by arbitrarily increasing $m^{+}$or $m^{-}$, we may make $\left|\tau^{\prime}\right|$ as large as we like. This $\tau^{\prime}$ is arbitrary and effective local potentials calculated from it are arbitrary. However, once again we emphasize that if we calculated the scattering with $V_{\tau}$ exactly, we would get the same answer to order $N^{-1}$ whatever the value of $m^{ \pm}$.

\section{A LOCAL $t$ MATRIX}

We avoid the difficulty of approximating a nonlocal potential by constructing a $\tau$ which is already local, i.e.,

$$
\tau\left(E, \mathbf{k}, \mathbf{k}_{0}\right) \equiv \tau\left(E, \mathbf{k}-\mathbf{k}_{0}\right) .
$$

This automatically leads to a local optical potential ${ }^{5}$ where

$$
V\left(E, \mathbf{k}, \mathbf{k}_{0}\right) \equiv V(E, \mathbf{q})=N F(q) \tau(E, \mathbf{q}),
$$

We write $\tau(E, \mathbf{q})$ as

$$
\mathrm{q}=\mathbf{k}-\mathbf{k}_{0} \text {. }
$$

$$
\begin{aligned}
\boldsymbol{\tau}(E, \mathbf{q})= & \int \exp (-i \mathbf{q} \cdot \mathbf{r}) \varphi_{E}(\boldsymbol{r}) d^{3} \boldsymbol{r} \\
& =\sum_{l}(2 l+1) P_{l}\left[1-\left(q^{2} / 2 k^{2}\right)\right] t_{l}\left(k_{E}, k_{E}\right) .
\end{aligned}
$$

This form has been studied in relationship to Regge poles and the function $\tau(E, \mathrm{q})$ is well defined. ${ }^{6}$ To correctly identify $\psi\left(\mathbf{k}_{E}, \mathbf{r}\right)$ with $\chi\left(\mathbf{k}_{E}, \mathbf{r}\right)$ in the region $r>a$, it is necessary that $\varphi(r)$ is zero for $r>a$. We may

5 The reader will note the similarity between this expression and that found by Glauber [see $R$. Glauber, in Lectures in Theoretical Physics, edited by W. E. Brittin et al. (Interscience Publishers, Inc., New York, 1958), Vol. 1.

${ }^{6}$ See, e.g., R. C. Newton, The Complex $j$-Plane (W. A. Benjamin, Inc., New York, 1964). show that this is the case for a nonsingular potential $v(\boldsymbol{r})$ by noting that for sufficiently large $l$ we may use the Born approximation to determine $t_{l}\left(k_{E}, k_{E}\right)$. Thus,

$$
\left(-2 m / 4 \pi \hbar^{2}\right) \int j l^{2}\left(k_{E} r\right) v(r) r^{2} d r=\int j l^{2}\left(k_{E} r\right) \varphi(r) r^{2} d r,
$$

for all $l$ sufficiently large. ${ }^{4}$ This implies that

$$
\varphi(r)=-\left(2 m / 4 \pi \hbar^{2}\right) v(r)
$$

in the region near $r=a$, hence the result. ${ }^{7}$

We have not been able to give a convincing proof that $\tau(E, q)$ is the only local $t$ matrix we can write down. However, it does not matter if it is not, since it is well defined in terms of the on-shell properties of $t$. Calculations with $V_{\boldsymbol{r}}$ are now straightforward.

\section{CONCLUSION}

To apply this theory to scattering from real nuclei we have to convince ourselves that consecutive collisions occur with nucleons that do not overlap. The constant density property of nuclei makes this statement valid to order $N^{-1}$, i.e., repulsive core correlations and the exclusion principle ensure that most nucleons are interacting with only one or two nearest neighbors at any one time. Recoil effects make no intrinsic difference to the conclusions drawn from scattering from potentials. ${ }^{3}$ However, we must, of course, correct the impulse approximation. ${ }^{8}$

As far as practical applications are concerned this theory removes a stumbling block noted by Reading and MacKellar ${ }^{8}$ in the $90-300-\mathrm{MeV}$ energy region for nucleon-nucleus scattering. There it was found that $t^{\prime}$ was very sensitive to the assumed effective mass $m^{*}$ of the struck target nucleon. Now we can ignore the nonlocality altogether. Its sensitivity no longer presents a problem. Finally, we note that the optical potential at small $r$ is sensitive to $\tau(E, q)$ at large $q$ and that $\tau(E, q)$ will be dominated by the Regge amplitude in that region. Hence, information on optical potentials for small $r$ may give information about Regge trajectories. This is not off-energy-shell information, since once all the phase shifts are known the Regge trajectory follows in principle, though not necessarily in practice.

\section{ACKNOWLEDGMENTS}

We wish to thank Professor R. E. Peierls for a useful discussion which prompted this work, and Dr. J. K. Perring for many valuable suggestions.

\footnotetext{
${ }^{7}$ We have, of course, that $\varphi(r)$ is independent of $l$.

8 J. F. Reading and A. D. MacKellar, Phys. Rev. 173, 1026 (1968).
} 